\title{
Research Article \\ Global Stability of a SLIT TB Model with Staged Progression
}

\author{
Yakui Xue and Xiaohong Wang \\ Department of Mathematics, North University of China, Shanxi, Taiyuan 030051, China \\ Correspondence should be addressed to Yakui Xue, xyk5152@163.com
}

Received 14 January 2012; Revised 28 July 2012; Accepted 16 August 2012

Academic Editor: Vu Phat

Copyright (C) 2012 Y. Xue and X. Wang. This is an open access article distributed under the Creative Commons Attribution License, which permits unrestricted use, distribution, and reproduction in any medium, provided the original work is properly cited.

Because the latent period and the infectious period of tuberculosis (TB) are very long, it is not reasonable to consider the time as constant. So this paper formulates a mathematical model that divides the latent period and the infectious period into n-stages. For a general n-stage stage progression (SP) model with bilinear incidence, we analyze its dynamic behavior. First, we give the basic reproduction number $R_{0}$. Moreover, if $R_{0} \leq 1$, the disease-free equilibrium $P_{0}$ is globally asymptotically stable and the disease always dies out. If $R_{0}>1$, the unique endemic equilibrium $P^{*}$ is globally asymptotically stable and the disease persists at the endemic equilibrium.

\section{Introduction}

Tuberculosis (TB) is one of the oldest recorded diseases of mankind. It is an disease caused by the infection of bacterium Mycobacterium tuberculosis. The disease is most commonly transmitted from a person suffering from infectious (active) tuberculosis to other persons by infected droplets created when the person with active TB coughs or sneezes. Most infected people do not develop the progressive disease. When the first time after being infected with Mycobacterium tuberculosis, the individual generally will experience a latent phase. TB progresses through a long latent period and a long infectious period. For this case, the infection can vary greatly in time. The progression of a typical TB infection can take four weeks to several years before the development into active disease, and a few individuals directly become infectious without experiencing latency. Moreover, most infected people do not develop the active disease in his or her life. In the infectious period, individual differences lead to different course. The longest infectious period is several decades while the shortest maybe only a few months. Moreover, the treatment of TB has side effects (sometimes quite serious) and takes varying time depending on the other various factors such as nutritional status and/or access to decent medical care and living conditions [1]. The progression of a TB infection goes through several distinct stages. Similarly, HIV virus has the long incubation 
and infectious periods (infection age, from 8 to 10 years). During the incubation period, the infectivity of infected people is varying depending on the time since infection. When symptom onset appears, AIDS population transmission rate depends on disease age (i.e., the time elapsed since the onset) [2]. Different from common infectious diseases, the time scale of TB or HIV / AIDS transmission is so long that demographic of the host population could affect transmission process. The classic compartmental models that postulate all the hosts are similar and imprecise to describe the spread of an infection. For explore the issue, many authors formulate staged progression (SP) models [1, 3-9] and delayed epidemic models [10]. In [5], the authors analyze a mathematical model for infectious diseases that progress through distinct stages within infected hosts and prove the global dynamics of the equilibria. Hyman et al. [4] created two SP models to show the impact of variations in infectiousness. In [10], the author formulate a delayed SIR epidemic model by introducing a latent period into susceptible and infectious individuals in incidence rate. Mathematical modeling has become an important tool in analyzing the epidemiological characteristics of infectious diseases and can provide useful control measures. In [11-18], several variants and generalizations of the Beverton CHolt model (standard time-invariant, time-varying parameterized, generalized model or modified generalized model) have been investigated at the levels of stability, cycleoscillatory behavior, permanence, and control through the manipulation of the carrying capacity. De la Sen et al. studied the impact of vaccination for infectious diseases. This paper considers the latent period and the infectious period to formulate a n-stage SP model with bilinear incidence (based on the model in [3]).

To formulate an SP model, the host population is divided into the following epidemiological classes or subgroups: the susceptible compartment $S$; the latent compartment $L_{i}$ (infected but not infectious), whose members are in the $i$ th stage of the disease progression, where $i=1,2, \ldots, n$; the infectious compartment $I_{j}$, whose members are in the $j$ th stage of the disease progression, where $j=1,2, \ldots, m$; the treated compartment $T$. $N$ denotes the total population. Here we assume that the latent period is averagely divided into $n$ stages and the infectious period is averagely divided into $m$ stages. We also assume that hosts in the treated compartment are noninfectious due to inactivity. Using Figure 1, we formulate the following model:

$$
\begin{gathered}
\frac{d S}{d t}=\Lambda-\lambda S-d S, \\
\frac{d L_{1}}{d t}=\lambda S-(n \delta+d) L_{1}, \\
\frac{d L_{i}}{d t}=n \delta L_{i-1}-(n \delta+d) L_{i}, \quad i=2,3, \ldots, n, \\
\frac{d I_{1}}{d t}=n \delta L_{n}-(m \gamma+\mu+d) I_{1}, \\
\frac{d I_{j}}{d t}=m \gamma I_{j-1}-(m \gamma+\mu+d) I_{j}, \quad j=2,3, \ldots, m-1, \\
\frac{d I_{m}}{d t}=m \gamma I_{m-1}-(k+\mu+d) I_{m}, \\
\frac{d T}{d t}=k I_{m}-d T .
\end{gathered}
$$




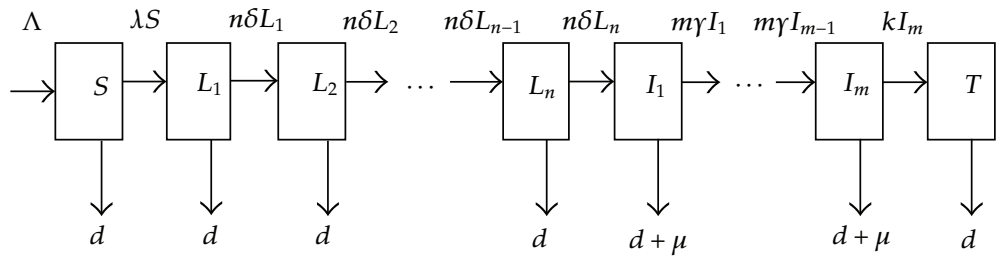

Figure 1: Transfer diagram.

The incidence form is $\lambda S$, where the force of infection

$$
\lambda=\sum_{j=1}^{m} \lambda_{j} I_{j}
$$

is the bilinear incidence; $1 / \delta$ is the incubation period, and $1 / n \delta$ is the average incubation period from the $i$ th stage to $(i+1)$ th stage, for $i=1,2, \ldots, n-1 ; 1 / \gamma$ is the infectious period, and $1 / m \gamma$ is the average infectious period from the $j$ th stage to $(j+1)$ th stage, for $j=1,2, \ldots, m-$ $2 ; \Lambda$ is the recruitment rate of susceptible; $k$ is per-capita treatment rates, respectively; $d$ is the per-capita natural death rate (and hence $1 / d$ is an average lifespan of the healthy individuals in the population); $\mu$ is the per capita disease-induced death rate. $\Lambda, \delta, d, k, \gamma, \mu, \lambda$ are positive constants. Because in the model (1.1), the first $n+m+1$ equations do not contain variable $T$, so dynamical behaviors of the model (1.1) are equivalent to the model (1.3).

$$
\begin{gathered}
\frac{d S}{d t}=\Lambda-\lambda S-d S, \\
\frac{d L_{1}}{d t}=\lambda S-(n \delta+d) L_{1}, \\
\frac{d L_{i}}{d t}=n \delta L_{i-1}-(n \delta+d) L_{i}, \quad i=2,3, \ldots, n, \\
\frac{d I_{1}}{d t}=n \delta L_{n}-(m \gamma+\mu+d) I_{1}, \\
\frac{d I_{j}}{d t}=m \gamma I_{j-1}-(m \gamma+\mu+d) I_{j}, \quad j=2,3, \ldots, m-1, \\
\frac{d I_{m}}{d t}=m \gamma I_{m-1}-(k+\mu+d) I_{m} .
\end{gathered}
$$

Let $N=S+\sum_{i=1}^{n} L_{i}+\sum_{j=1}^{m} I_{j}$, then using (1.3) we have

$$
\frac{d N}{d t}=\Lambda-d N-\mu \sum_{j=1}^{m} I_{j}-k I_{m}
$$


This implies that $\lim _{t \rightarrow \infty} \sup N(t) \leq \Lambda / d$. Therefore, the model can be studied in the feasible region

$$
\Gamma=\left\{\left(S, L_{1}, \ldots, L_{n}, I_{1}, \ldots, I_{m}\right) \in R_{n+m+1}^{+}: 0 \leq S+L_{1}+\cdots+L_{n}+I_{1}+\cdots+I_{m} \leq \frac{\Lambda}{d}\right\} .
$$

\section{The Basic Reproduction Number}

The disease-free equilibrium is obtained by setting the right side of each of the $n+m+1$ differential equations equal to zero in system (1.3).

If $I_{j}=0, j=1, \ldots, m$, it is easy to deduce the disease-free equilibrium as follows:

$$
P_{0}=\left(\frac{\Lambda}{d}, 0, \ldots, 0,0,0, \ldots, 0\right)
$$

Next, we derive the basic reproductive number of (1.3) by the method of nextgeneration matrix formulated in [19].

Let $x=\left(L_{1}, L_{2}, \ldots, L_{n}, I_{1}, I_{2}, \ldots, I_{m}\right)^{T}$. Then the last $n+m$ equations of model (1.3) can be written as

$$
x^{\prime}=F(x)-\mho(x)
$$

where

$$
\left.\begin{array}{c}
f(x)=\left(\begin{array}{c}
\lambda S \\
0 \\
\vdots \\
0 \\
0 \\
0 \\
\vdots \\
0
\end{array}\right), \\
(n \delta+d) L_{1} \\
\vdots \\
-n \delta L_{1}+(n \delta+d) L_{2} \\
-n \delta L_{n-1}+(n \delta+d) L_{n} \\
-n \delta L_{n}+(m \gamma+\mu+d) I_{1} \\
-m \gamma I_{1}+(m \gamma+\mu+d) I_{2} \\
\vdots \\
-m \gamma I_{m-1}+(k+\mu+d) I_{m}
\end{array}\right) .
$$


By calculating the Jacobian matrices of $\mathcal{F}(x)$ and $\mho(x)$ at the disease-free equilibrium $P_{0}$, we have

$$
\begin{aligned}
& F=\left(\begin{array}{cccccccc}
0 & 0 & \cdots & 0 & \lambda_{1} S & \lambda_{2} S & \cdots & \lambda_{m} S \\
0 & 0 & \cdots & 0 & 0 & 0 & \cdots & 0 \\
\vdots & \vdots & \vdots & \vdots & \vdots & \vdots & \vdots & \vdots \\
0 & 0 & \cdots & 0 & 0 & 0 & \cdots & 0 \\
0 & 0 & \cdots & 0 & 0 & 0 & \cdots & 0 \\
0 & 0 & \cdots & 0 & 0 & 0 & \cdots & 0 \\
\vdots & \vdots & \vdots & \vdots & \vdots & \vdots & \vdots & \vdots \\
0 & 0 & \cdots & 0 & 0 & 0 & \cdots & 0
\end{array}\right) \\
& V=\left(\begin{array}{cccccccc}
n \delta+d & 0 & \cdots & 0 & 0 & 0 & \cdots & 0 \\
-n \delta & n \delta+d & \cdots & 0 & 0 & 0 & \cdots & 0 \\
\vdots & \vdots & \vdots & \vdots & \vdots & \vdots & \vdots & \vdots \\
0 & 0 & \cdots & n \delta+d & 0 & 0 & \cdots & 0 \\
0 & 0 & \cdots & -n \delta & m \gamma+\mu+d & 0 & \cdots & 0 \\
0 & 0 & \cdots & 0 & -m \gamma & m \gamma+\mu+d & \cdots & 0 \\
\vdots & \vdots & \vdots & \vdots & \vdots & \vdots & \vdots & \vdots \\
0 & 0 & \cdots & 0 & 0 & 0 & \cdots & k+\mu+d
\end{array}\right) .
\end{aligned}
$$

Let $I_{(n+m) \times(n+m)}$ be the identity matrix. Solving the matrix equation $V X=I_{(n+m) \times(n+m)}$, we obtain that $X=V^{-1}$ is a lower triangular matrix

$$
\begin{aligned}
& V^{-1}=\left(\begin{array}{cccccccc}
c_{11} & 0 & \cdots & 0 & 0 & 0 & \cdots & 0 \\
c_{21} & c_{22} & \cdots & 0 & 0 & 0 & \cdots & 0 \\
\vdots & \vdots & \vdots & \vdots & \vdots & \vdots & \vdots & \vdots \\
c_{n 1} & c_{n 2} & \cdots & c_{n n} & 0 & 0 & \cdots & 0 \\
c_{n+1,1} & c_{n+1,2} & \cdots & c_{n+1, n} & c_{n+1, n+1} & 0 & \cdots & 0 \\
c_{n+2,1} & c_{n+2,2} & \cdots & c_{n+2, n} & c_{n+2, n+1} & c_{n+2, n+2} & \cdots & 0 \\
\vdots & \vdots & \vdots & \vdots & \vdots & \vdots & \vdots & \vdots \\
c_{n+m, 1} & c_{n+m, 2} & \cdots & c_{n+m, n} & c_{n+m, n+1} & c_{n+m, n+2} & \cdots & c_{n+m, n+m}
\end{array}\right) \\
&=\left(\begin{array}{ll}
A_{1} & A_{2} \\
A_{3} & A_{4}
\end{array}\right),
\end{aligned}
$$


where

$$
\begin{aligned}
& A_{1}=\left(\begin{array}{cccc}
c_{11} & 0 & \cdots & 0 \\
c_{21} & c_{22} & \cdots & 0 \\
\vdots & \vdots & \vdots & \vdots \\
c_{n 1} & c_{n 2} & \cdots & c_{n n}
\end{array}\right)=\left(\begin{array}{cccc}
\frac{1}{n \delta+d} & 0 & \cdots & 0 \\
\frac{n \delta}{(n \delta+d)^{2}} & \frac{1}{n \delta+d} & \cdots & 0 \\
\vdots & \vdots & \vdots & \vdots \\
\frac{(n \delta)^{n-1}}{(n \delta+d)^{n}} & \frac{(n \delta)^{n-2}}{(n \delta+d)^{n-1}} & \cdots & \frac{1}{n \delta+d}
\end{array}\right) \\
& A_{2}=\left(\begin{array}{cccc}
0 & 0 & \cdots & 0 \\
0 & 0 & \cdots & 0 \\
\vdots & \vdots & \vdots & \vdots \\
0 & 0 & \cdots & 0
\end{array}\right) \\
& A_{3}=\left(\begin{array}{cccc}
c_{n+1,1} & c_{n+1,2} & \cdots & c_{n+1, n} \\
c_{n+2,1} & c_{n+2,2} & \cdots & c_{n+2, n} \\
\vdots & \vdots & \vdots & \vdots \\
c_{n+m, 1} & c_{n+m, 2} & \cdots & c_{n+m, n}
\end{array}\right) \\
& =\left(\begin{array}{cccc}
\frac{(n \delta)^{n}}{(m \gamma+\mu+d)(n \delta+d)^{n}} & \frac{(n \delta)^{n-1}}{(m \gamma+\mu+d)(n \delta+d)^{n-1}} & \cdots & \frac{n \delta}{(m \gamma+\mu+d)(n \delta+d)} \\
\frac{(n \delta)^{n}(m \gamma)}{(m \gamma+\mu+d)^{2}(n \delta+d)^{n}} & \frac{(n \delta)^{n-1}(m \gamma)}{(m \gamma+\mu+d)^{2}(n \delta+d)^{n-1}} & \cdots & \frac{(n \delta)(m \gamma)}{(m \gamma+\mu+d)^{2}(n \delta+d)} \\
\vdots & \vdots & \vdots & \vdots \\
\frac{(n \delta)^{n}(m \gamma)^{m-1}}{A(n \delta+d)^{n}(k+\mu+d)} & \frac{(n \delta)^{n-1}(m \gamma)^{m-1}}{A(n \delta+d)^{n-1}(k+\mu+d)} & \cdots & \frac{(n \delta)(m \gamma)^{m-1}}{A(n \delta+d)(k+\mu+d)}
\end{array}\right) \\
& A_{4}=\left(\begin{array}{cccc}
c_{n+1, n+1} & 0 & \cdots & 0 \\
c_{n+2, n+1} & c_{n+2, n+2} & \cdots & 0 \\
\vdots & \vdots & \vdots & \vdots \\
c_{n+m, n+1} & c_{n+m, n+2} & \cdots & c_{n+m, n+m}
\end{array}\right) \\
& =\left(\begin{array}{cccc}
\frac{1}{m \gamma+\mu+d} & 0 & \cdots & 0 \\
\frac{m \gamma}{(m \gamma+\mu+d)^{2}} & \frac{1}{m \gamma+\mu+d} & \cdots & 0 \\
\vdots & \vdots & \vdots & \vdots \\
\frac{(m \gamma)^{m-1}}{A(k+\mu+d)} & \frac{(m \gamma)^{m-2}}{(m \gamma+\mu+d)^{m-2}(k+\mu+d)} & \cdots & \frac{1}{k+\mu+d}
\end{array}\right) \text {, }
\end{aligned}
$$

where $\mathcal{A}$ equals $(m \gamma+\mu+d)^{m-1}$. 
$F V^{-1}$ is the next-generation matrix for model (1.3). It then follows that the spectral radius of matrix $F V^{-1}$ is

$$
\begin{aligned}
\rho\left(F V^{-1}\right)= & \frac{\left(\lambda_{1} \Lambda / d\right)(n \delta)^{n}}{(m \gamma+\mu+d)(n \delta+d)^{n}}+\frac{\left(\lambda_{2} \Lambda / d\right)(n \delta)^{n}(m \gamma)}{(m \gamma+\mu+d)^{2}(n \delta+d)^{n}} \\
& +\cdots+\frac{\left(\lambda_{m} \Lambda / d\right)(n \delta)^{n}(m \gamma)^{m-1}}{(m \gamma+\mu+d)^{m-1}(n \delta+d)^{n}(k+\mu+d)} .
\end{aligned}
$$

According to Theorem 2 in [19], the basic reproduction number of model (1.3) is

$$
\begin{aligned}
R_{0}= & \frac{\left(\lambda_{1} \Lambda / d\right)(n \delta)^{n}}{(m \gamma+\mu+d)(n \delta+d)^{n}}+\frac{\left(\lambda_{2} \Lambda / d\right)(n \delta)^{n}(m \gamma)}{(m \gamma+\mu+d)^{2}(n \delta+d)^{n}} \\
& +\cdots+\frac{\left(\lambda_{m} \Lambda / d\right)(n \delta)^{n}(m \gamma)^{m-1}}{(m \gamma+\mu+d)^{m-1}(n \delta+d)^{n}(k+\mu+d)}
\end{aligned}
$$

Therefore, $R_{0}$ gives the number of secondary infectious cases produced by an infectious individual during his or her effective infectious period when introduced in a population of susceptible. If $R_{0}>1$, then $P_{0}$ becomes unstable and the disease becomes endemic. Moreover, a unique endemic equilibrium $P^{*}=\left(S^{*}, L_{1}^{*}, L_{2}^{*}, \ldots, L_{n}^{*}, I_{1}^{*}, I_{2}^{*}, \ldots, I_{m}^{*}\right)$ exists in the interior of $\Gamma$. Next, we prove the uniqueness of the endemic equilibrium when $R_{0}>1$.

\section{Equilibria}

An equilibrium $\left(S, L_{1}, L_{2}, \ldots, L_{n}, I_{1}, I_{2}, \ldots, I_{m}\right)$ of (1.3) satisfies

$$
\begin{gathered}
0=\Lambda-\lambda S-d S, \\
0=\lambda S-(n \delta+d) L_{1}, \\
0=n \delta L_{i-1}-(n \delta+d) L_{i}, \quad i=2,3, \ldots, n, \\
0=n \delta L_{n}-(m \gamma+\mu+d) I_{1}, \\
0=m \gamma I_{j-1}-(m \gamma+\mu+d) I_{j}, \quad j=2,3, \ldots, m-1, \\
0=m \gamma I_{m-1}-(k+\mu+d) I_{m},
\end{gathered}
$$

where $\lambda$ is given in Section 1 . The disease-free equilibrium $P_{0}=(\Lambda / d, 0,0, \ldots, 0,0,0, \ldots, 0)$ exists for all positive parameter values. Next we consider the existence of endemic equilibria $P^{*}=\left(S^{*}, L_{1}^{*}, L_{2}^{*}, \ldots, L_{n}^{*}, I_{1}^{*}, I_{2}^{*}, \ldots, I_{m}^{*}\right), S^{*}>0, L_{i}^{*}>0, I_{j}^{*}>0, i=1, \ldots, n, j=1, \ldots, m$. First, we introduce the definition and properties of M-matrices. Most of the texts on matrix theory can find them [20]. 
Definition 3.1. $B_{n \times n}$ is a M-matrix if

(1) Off-diagonal entries of $B$ are nonpositive, and

(2) $B$ is positively stable, namely, all eigenvalues of $B$ have positive real parts.

Proposition 3.2. Properties of M-matrices

(1) $B=\alpha I-P, P \geq 0, \alpha>\rho(P)$, the spectral radius of $P$.

(2) $B$ is nonsingular and $B^{-1} \geq 0$.

(3) There exists $\beta>0$ such that $B^{-1} x \geq \beta x$ for $x \geq 0$.

According to the above, we know that

$$
V=\left(\begin{array}{cccccccc}
n \delta+d & 0 & \cdots & 0 & 0 & 0 & \cdots & 0 \\
-n \delta & n \delta+d & \cdots & 0 & 0 & 0 & \cdots & 0 \\
\vdots & \vdots & \vdots & \vdots & \vdots & \vdots & \vdots & \vdots \\
0 & 0 & \cdots & n \delta+d & 0 & 0 & \cdots & 0 \\
0 & 0 & \cdots & -n \delta & m \gamma+\mu+d & 0 & \cdots & 0 \\
0 & 0 & \cdots & 0 & -m \gamma & m \gamma+\mu+d & \cdots & 0 \\
\vdots & \vdots & \vdots & \vdots & \vdots & \vdots & \vdots & \vdots \\
0 & 0 & \cdots & 0 & 0 & 0 & \cdots & k+\mu+d
\end{array}\right)
$$

Proposition 3.3. The following holds for the matrix $V$ defined above.

(1) $V$ is a M-matrix.

(2) $V^{-1}$ exists and is a nonnegative matrix.

(3) There exists $v>0$ such that $V^{-1} x \geq v x$ for $x \geq 0$.

By Proposition 3.3, we know that

$$
v=\left(0,0, \ldots, 0, \lambda_{1}, \lambda_{2}, \ldots, \lambda_{m}\right) V^{-1}\left(\begin{array}{c}
1 \\
0 \\
\vdots \\
0 \\
0 \\
0 \\
\vdots \\
0
\end{array}\right)>0
$$

Then, we obtain the result. 
Journal of Applied Mathematics

Theorem 3.4. If $R_{0} \leq 1$, then $P_{0}$ is the only equilibrium in $\Gamma$. If $R_{0}>1$, then a unique endemic equilibrium $P^{*}$ exists in the interior of $\Gamma$.

Proof. The last $n+m$ equations of (3.1) can be written in the form

$$
V\left(\begin{array}{c}
L_{1} \\
L_{2} \\
\vdots \\
L_{n} \\
I_{1} \\
I_{2} \\
\vdots \\
I_{m}
\end{array}\right)=\left(\begin{array}{c}
\lambda S \\
0 \\
\vdots \\
0 \\
0 \\
0 \\
\vdots \\
0
\end{array}\right)
$$

or

$$
\left(\begin{array}{c}
L_{1} \\
L_{2} \\
\vdots \\
L_{n} \\
I_{1} \\
I_{2} \\
\vdots \\
I_{m}
\end{array}\right)=V^{-1}\left(\begin{array}{c}
\lambda S \\
0 \\
\vdots \\
0 \\
0 \\
0 \\
\vdots \\
0
\end{array}\right)
$$

Multiplying the row vector $\left(0,0, \ldots, 0, \lambda_{1}, \lambda_{2}, \ldots, \lambda_{m}\right)$ to (3.5), we have

$$
\sum_{j=1}^{m} \lambda_{j} I_{j}=\left(0,0, \ldots, 0, \lambda_{1}, \lambda_{2}, \ldots, \lambda_{m}\right)\left(\begin{array}{c}
L_{1} \\
L_{2} \\
\vdots \\
L_{n} \\
I_{1} \\
I_{2} \\
\vdots \\
I_{m}
\end{array}\right)=\left(0,0, \ldots, 0, \lambda_{1}, \lambda_{2}, \ldots, \lambda_{m}\right) V^{-1}\left(\begin{array}{c}
\lambda S \\
0 \\
\vdots \\
0 \\
0 \\
0 \\
\vdots \\
0
\end{array}\right)
$$


Using $\lambda=\sum_{j=1}^{m} \lambda_{j} I_{j}$, we obtain

$$
\begin{aligned}
& \sum_{j=1}^{m} \lambda_{j} I_{j}=\left(0,0, \ldots, 0, \lambda_{1}, \lambda_{2}, \ldots, \lambda_{m}\right) V^{-1}\left(\begin{array}{c}
\lambda S \\
0 \\
\vdots \\
0 \\
0 \\
0 \\
\vdots \\
0
\end{array}\right) \\
& =\left(0,0, \ldots, 0, \lambda_{1}, \lambda_{2}, \ldots, \lambda_{m}\right) V^{-1}\left(\begin{array}{c}
S \\
0 \\
\vdots \\
0 \\
0 \\
0 \\
\vdots \\
0
\end{array}\right) \sum_{j=1}^{m} \lambda_{j} I_{j} .
\end{aligned}
$$

Because $\sum_{j=1}^{m} \lambda_{j} I_{j} \neq 0$, then

$$
1=\left(0,0, \ldots, 0, \lambda_{1}, \lambda_{2}, \ldots, \lambda_{m}\right) V^{-1}\left(\begin{array}{c}
S \\
0 \\
\vdots \\
0 \\
0 \\
0 \\
\vdots \\
0
\end{array}\right)=\left(0,0, \ldots, 0, \lambda_{1}, \lambda_{2}, \ldots, \lambda_{m}\right) V^{-1} S\left(\begin{array}{c}
1 \\
0 \\
\vdots \\
0 \\
0 \\
0 \\
\vdots \\
0
\end{array}\right)=v S .
$$

It is clear that the equation $v S=1$ has a unique positive solution $S^{*}=1 / v$ in the interval $(0, \Lambda / d)$ when $R_{0}>1$. Substitute $S^{*}=1 / v$ into $(3.1)$, and we obtain $L_{1}^{*}, \ldots, L_{n}^{*}, I_{1}^{*}, \ldots, I_{n}^{*}$ are unique positive solution. So the endemic equilibrium $P^{*}$ is unique when $R_{0}>1$.

\section{Stability of the Equilibria}

In this section, we employ the direct Lyapunov method with a Lyapunov function of the form

$$
\sum A_{i}\left(X_{i}(t)-X_{i}^{*} \ln X(t)\right)
$$

where $A_{i}$ is a properly selected constant, $X_{i}$ is the population of the $i$ th compartment, and $X_{i}^{*}$ is the equilibrium level, to study properties of this model. This function is referenced in 
many papers [21-23], including the models with multiple parallel infectious stages [4, 24] and models with nonlinear incidence rates of different forms [25-28].

Now we are ready to proceed to the global properties of the model.

Theorem 4.1. If $R_{0} \leq 1$, the disease-free equilibrium $P_{0}=(\Lambda / d, 0,0, \ldots, 0,0,0, \ldots, 0)$ is globally asymptotically stable in $\Gamma$.

Proof. Consider the function

$$
W=\sum_{i=1}^{n} A_{i} L_{i}+\sum_{j=1}^{m} B_{j} I_{j}
$$

where $A_{i}$ and $B_{j}(i=1,2, \ldots, n-1 ; j=1,2, \ldots, m-2 ;)$ are properly selected constants. Their values are as follows:

$$
\begin{gathered}
A_{1}=(n \delta)^{n}(m \gamma)^{m-1}>0, \\
A_{2}=(n \delta)^{n-1}(n \delta+d)(m \gamma)^{m-1}>0, \\
A_{3}=(n \delta)^{n-2}(n \delta+d)^{2}(m \gamma)^{m-1}>0, \\
\vdots \\
A_{n}=(n \delta)(n \delta+d)^{n-1}(m \gamma)^{m-1}>0, \\
B_{1}=(n \delta+d)^{n}(m \gamma)^{m-1}>0, \\
B_{2}=-\left[(n \delta)^{n}(m \gamma)^{m-2}\left(\lambda_{1} S\right)-(n \delta+d)^{n}(m \gamma)^{m-2}(m \gamma+\mu+d)\right] \\
B_{3}=-\left[(n \delta)^{n}(m \gamma)^{m-2}\left(\lambda_{2} S\right)+(n \delta)^{n}(m \gamma)^{m-3}(m \gamma+\mu+d)\left(\lambda_{1} S\right)\right. \\
\left.-(n \delta+d)^{n}(m \gamma)^{m-3}(m \gamma+\mu+d)^{2}\right], \\
B_{m-1}=-\left[\begin{array}{c}
(n \delta)^{n}(m \gamma)^{m-2}\left(\lambda_{m-2} S\right)^{2}+(n \delta)^{n}(m \gamma)^{m-3}(m \gamma+\mu+d)\left(\lambda_{m-3} S\right) \\
\left.+\cdots+(n \delta)^{n}(m \gamma)(m \gamma+\mu+d)^{m-3}\left(\lambda_{1} S\right)-(n \delta+d)^{n}(m \gamma)(m \gamma+\mu+d)^{m-2}\right], \\
B_{m}=-\left[(n \delta)^{n}(m \gamma)^{m-2}\left(\lambda_{m-1} S\right)+(n \delta)^{n}(m \gamma)^{m-3}(m \gamma+\mu+d)\left(\lambda_{m-2} S\right)\right. \\
\left.+\cdots+(n \delta)^{n}(m \gamma+\mu+d)^{m-2}\left(\lambda_{1} S\right)-(n \delta+d)^{n}(m \gamma+\mu+d)^{m-1}\right] .
\end{array}\right.
\end{gathered}
$$

Because $R_{0} \leq 1$, the each part of $R_{0}$ should be less than 1, that is, $\left(\lambda_{1} \Lambda / d\right)(n \delta)^{n}<$ $(m \gamma+\mu+d)(n \delta+d)^{n},\left(\lambda_{2} \Lambda / d\right)(n \delta)^{n}(m \gamma)<(m \gamma+\mu+d)^{2}(n \delta+d)^{n}, \ldots,\left(\lambda_{m} \Lambda / d\right)(n \delta)^{n}(m \gamma)^{m-1}<$ 
$(m \gamma+\mu+d)^{m-1}(n \delta+d)^{n}(k+\mu+d)$. Therefore, we can obtain that $B_{2}, B_{3}, \ldots, B_{m}$ are greater than 0 . This function is defined and continuous for all $L_{i}, I_{j}>0$.

Its derivative along the solutions to the system (1.3) is

$$
\begin{aligned}
\dot{W}= & A_{1} \dot{L}_{1}+\cdots+A_{n} \dot{L}_{n}+B_{1} \dot{I}_{1}+\cdots+B_{m} \dot{I}_{m}=\left[(n \delta)^{n}(m \gamma)^{m-1}\right]\left[\sum_{j=1}^{m} \lambda_{j} I_{j} S-(n \delta+d) L_{1}\right] \\
& +\left[(n \delta)^{n-1}(n \delta+d)(m \gamma)^{m-1}\right]\left[n \delta L_{1}-(n \delta+d) L_{2}\right] \\
& +\cdots+\left[(n \delta)(n \delta+d)^{n-1}(m \gamma)^{m-1}\right]\left[n \delta L_{n-1}-(n \delta+d) L_{n}\right] \\
& +\left[(n \delta+d)^{n}(m \gamma)^{m-1}\right]\left[(n \delta+d) L_{n}-(m \gamma+\mu+d) I_{1}\right]-\left[(n \delta)^{n}(m \gamma)^{m-2}\left(\lambda_{1} S\right)\right. \\
& \left.\quad-(n \delta+d)^{n}(m \gamma)^{m-2}(m \gamma+\mu+d)\right]\left[m \gamma I_{1}-(m \gamma+\mu+d) I_{2}\right]-\cdots \\
& -\left[(n \delta)^{n}(m \gamma)^{m-2}\left(\lambda_{m-1} S\right)+(n \delta)^{n}(m \gamma)^{m-3}(m \gamma+\mu+d)\left(\lambda_{m-2} S\right)+\cdots\right. \\
& \left.+(n \delta)^{n}(m \gamma+\mu+d)^{m-2}\left(\lambda_{1} S\right)-(n \delta+d)^{n}(m \gamma+\mu+d)^{m-1}\right]\left[m \gamma I_{m-1}-(k+\mu+d) I_{m}\right] \\
= & I_{m}\left[(n \delta)^{n}(m \gamma)^{m-1}\left(\lambda_{m} S\right)+(n \delta)^{n}(m \gamma)^{m-2}(k+\mu+d)\left(\lambda_{m-1} S\right)\right. \\
& \left.+\cdots+(n \delta)^{n}(m \gamma+\mu+d)^{m-2}(k+\mu+d)\left(\lambda_{1} S\right)-(n \delta+d)^{n}(m \gamma+\mu+d)^{m-1}(k+\mu+d)\right] \\
= & I_{m}(n \delta+d)^{n}(m \gamma+\mu+d)^{m}\left(R_{0}-1\right) .
\end{aligned}
$$

If $R_{0} \leq 1$, then $\dot{W} \leq 0$. Note that, $\dot{W}=0$ only if $I_{m}=0$. The maximum invariant set in $G=\left\{\left(S, L_{1}, \ldots, L_{n}, I_{1}, \ldots, I_{m}\right): \dot{W}=0\right\}$ is the singleton $P_{0}$. The global stability of $P_{0}$ when $R_{0} \leq 1$ follows from LaSalle's invariance principle [29]. The global attractivity of $P_{0}$ and the Lyapunov function $W$ imply that $P_{0}$ is also locally stable, since otherwise $P_{0}$ will have a homoclinic orbit that is entirely contained in $G$, contradicting that the largest compact invariant set in $G$ is $P_{0}$. This establishes the global stability of $P_{0}$ when $R_{0} \leq 1$.

Theorem 4.2. If $R_{0}>1$, the endemic equilibrium $P^{*}=\left(S^{*}, L_{1}^{*}, L_{2}^{*}, \ldots, L_{n}^{*}, I_{1}^{*}, \ldots, I_{n}^{*}\right)$ is globally asymptotically stable in $\Gamma$.

Proof. Let us consider the function

$$
\begin{aligned}
V= & C\left(S-S^{*} \ln S\right)+D\left(L_{1}-L_{1}^{*} \ln L_{1}\right)+\sum_{i=2}^{n} E_{i}\left(L_{i}-L_{i}^{*} \ln L_{i}\right) \\
& +F\left(I_{1}-I_{1}^{*} \ln I_{1}\right)+\sum_{j=2}^{m-1} G_{j}\left(I_{j}-I_{j}^{*} \ln I_{j}\right)+H\left(I_{m}-I_{m}^{*} \ln I_{m}\right),
\end{aligned}
$$


Journal of Applied Mathematics

where

$$
C=D=1, \quad E_{i}=\frac{\lambda S^{*}}{n \delta L_{i-1}^{*}}, \quad F=\frac{\lambda S^{*}}{n \delta L_{n}^{*}}, \quad G_{j}=\frac{\lambda S^{*}}{m \gamma I_{i-1}^{*}}, \quad H=\frac{\lambda S^{*}}{m \gamma I_{m-1}^{*}} .
$$

This function is defined and continuous for all $S, L_{i}, I_{j}>0$. By compute the derivative of $V$ along the solutions to the system (1.3), it follows that

$$
\begin{aligned}
\dot{V}= & (\Lambda-\lambda S-d S)\left(1-\frac{S^{*}}{S}\right)+\left[\lambda S-(n \delta+d) L_{1}\right]\left(1-\frac{L_{1}^{*}}{L_{1}}\right) \\
& +\sum_{i=2}^{n} \frac{\lambda S^{*}}{n \delta L_{i-1}^{*}}\left[n \delta L_{i-1}-(n \delta+d) L_{i}\right]\left(1-\frac{L_{i}^{*}}{L_{i}}\right) \\
& +\frac{\lambda S^{*}}{n \delta L_{n}^{*}}\left[n \delta L_{n}-(m \gamma+\mu+d) I_{1}\right]\left(1-\frac{I_{1}^{*}}{I_{1}}\right) \\
& +\sum_{j=2}^{m-1} \frac{\lambda S^{*}}{m \gamma I_{j-1}^{*}}\left[m \gamma I_{j-1}-(m \gamma+\mu+d) I_{j}\right]\left(1-\frac{I_{j}^{*}}{I_{j}}\right) \\
& +\frac{\lambda S^{*}}{m \gamma I_{m-1}^{*}}\left[m \gamma I_{m-1}-(k+\mu+d) I_{m}\right]\left(1-\frac{I_{m}^{*}}{I_{m}}\right) .
\end{aligned}
$$

Recalling that $\Lambda=\lambda S^{*}+d S^{*}, n \delta+d=\lambda S^{*} / L_{1}^{*}=n \delta L_{i-1}^{*} / L_{i}^{*}, m \gamma+\mu+d=n \delta L_{n}^{*} / I_{1}^{*}=$ $m \gamma I_{j-1}^{*} / I_{j}^{*}$, and that $k+\mu+d=m \gamma I_{m-1}^{*} / I_{m}^{*}$, we obtain

$$
\begin{aligned}
& \dot{V}=3 \lambda S^{*}+2 d S^{*}-d S-\frac{\lambda S^{* 2}}{S}-\frac{d S^{* 2}}{S}-\frac{L_{1} \lambda S^{*}}{L_{1}^{*}}-\frac{L_{1}^{*} \lambda S}{L_{1}} \\
& +\sum_{i=2}^{n}\left(\frac{L_{i-1} \lambda S^{*}}{L_{i-1}^{*}}-\frac{L_{i} \lambda S^{*}}{L_{i}^{*}}-\frac{L_{i-1} L_{i}^{*} \lambda S^{*}}{L_{i-1}^{*} L_{i}}+\lambda S^{*}\right) \\
& +\frac{L_{n} \lambda S^{*}}{L_{n}^{*}}-\frac{I_{1} \lambda S^{*}}{I_{1}^{*}}-\frac{L_{n} I_{1}^{*} \lambda S^{*}}{L_{n}^{*} I_{1}}+\lambda S^{*} \\
& +\sum_{j=2}^{m-1}\left(\frac{I_{j-1} \lambda S^{*}}{I_{j-1}^{*}}-\frac{I_{j} \lambda S^{*}}{I_{j}^{*}}-\frac{I_{j-1} I_{j}^{*} \lambda S^{*}}{I_{j-1}^{*} I_{j}}+\lambda S^{*}\right) \\
& +\frac{I_{m-1} \lambda S^{*}}{I_{m-1}^{*}}-\frac{I_{m} \lambda S^{*}}{I_{m}^{*}}-\frac{I_{m-1} I_{m}^{*} \lambda S^{*}}{I_{m-1}^{*} I_{m}}+\lambda S^{*} \\
& =d S^{*}\left(2-\frac{S^{*}}{S}-\frac{S}{S^{*}}\right)+\lambda S^{*}\left(n+m+2-\frac{S^{*}}{S}-\frac{L_{1}^{*} S}{L_{1} S^{*}}-\frac{L_{2}^{*} L_{1}}{L_{2} L_{1}^{*}}-\cdots\right. \\
& \left.-\frac{L_{n}^{*} L_{n-1}}{L_{n} L_{n-1}^{*}}-\frac{I_{1}^{*} L_{n}}{I_{1} L_{n}^{*}}-\frac{I_{1} I_{2}^{*}}{I_{2} I_{1}^{*}}-\cdots-\frac{I_{m-1} I_{m}^{*}}{I_{m} I_{m-1}^{*}}-\frac{I_{m}}{I_{m}^{*}}\right) .
\end{aligned}
$$


Applying the inequality

$$
\frac{a_{1}+a_{2}+\ldots+a_{n}}{n} \geq \sqrt[n]{a_{1} a_{2} \ldots a_{n}}, \quad \text { for } a_{i} \geq 0, i=1, \ldots, n,
$$

we have

$$
\dot{V} \leq 0 \text {. }
$$

Therefore, $d V / d t<0$ for all $S, L_{i}, I_{i}>0$, provided that $S^{*}, L_{i}^{*}, I_{i}^{*}$ are positive, where the equality $d V / d t=0$ holds only on the straight line $S=S^{*}, L_{i} / L_{i}^{*}=I_{i} / I_{i}^{*}$. It is easy to see that for both these systems, $P^{*}$ is the only equilibrium state on this line. Therefore, by LyapunovLaSalle asymptotic stability theorem [30,31], the positive equilibrium state $P^{*}$ is globally asymptotically stable in $\Gamma$.

\section{Conclusion}

According to the different length of the latent period and the infectious period of TB, in this paper, we proposed a general $n$-stage SP model with bilinear incidence to study the transmission dynamics of TB. What to do to make the results more accurate and tally with the actual situation. We prove that the global dynamics are completely determined by the basic reproduction number $R_{0}$. If $R_{0} \leq 1$, then the disease-free equilibrium $P_{0}$ is globally asymptotically stable and the disease always dies out. If $R_{0}>1$, the unique endemic equilibrium $P^{*}$ is globally asymptotically stable in the interior of the feasible region, and the disease persists at the endemic equilibrium.

\section{Acknowledgments}

The work is supported by the National Sciences Foundation of China (10471040) and the National Sciences Foundation of Shanxi Province (2009011005-1).

\section{References}

[1] B. R. Bloom, Tuberculosis: Pathogenesis, Protection, and Control, ASM Press, Washington, DC, USA, 1994.

[2] H. Liu and L. Li, "A class age-structured HIV/AIDS model with impulsive drug-treatment strategy," Discrete Dynamics in Nature and Society, vol. 2010, Article ID 758745, 2010.

[3] X. Wang and Y. Xue, "Global stability of a model with fast and slow Tuberculosis," Submitted.

[4] J. M. Hyman, J. Li, and E. Ann Stanley, "The differential infectivity and staged progression models for the transmission of HIV," Mathematical Biosciences, vol. 155, no. 2, pp. 77-109, 1999.

[5] H. Guo and M. Y. Li, "Global dynamics of a staged progression model for infectious diseases," Mathematical Biosciences and Engineering, vol. 3, no. 3, pp. 513-525, 2006.

[6] J. S. Koopman, J. A. Jacquez, G. W. Welch et al., "The role of early HIV infection in the spread of HIV through populations," Journal of Acquired Immune Deficiency Syndromes and Human Retrovirology, vol. 14, no. 3, pp. 249-258, 1997.

[7] A. L. Lloyd, "Destabilization of epidemic models with the inclusion of realistic distributions of infectious periods," Proceedings of the Royal Society B, vol. 268, no. 1470, pp. 985-993, 2001.

[8] A. L. Lloyd, "Realistic distributions of infectious periods in epidemic models: changing patterns of persistence and dynamics," Theoretical Population Biology, vol. 60, no. 1, pp. 59-71, 2001. 
[9] W. Y. Tan and Z. Ye, "Estimation of HIV infection and incubation via state space models," Mathematical Biosciences, vol. 167, no. 1, pp. 31-50, 2000.

[10] A. Kaddar, "Stability analysis in a delayed SIR epidemic model with a saturated incidence rate," Nonlinear Analysis. Modelling and Control, vol. 15, no. 3, pp. 299-306, 2010.

[11] M. De la Sen, "About the properties of a modified generalized Beverton-Holt equation in ecology models," Discrete Dynamics in Nature and Society, vol. 2008, Article ID 592950, p. 23, 2008.

[12] M. De la Sen, "The generalized Beverton-Holt equation and the control of populations," Applied Mathematical Modelling, vol. 32, no. 11, pp. 2312-2328, 2008.

[13] M. De la Sen and S. Alonso-Quesada, "A control theory point of view on Beverton-Holt equation in population dynamics and some of its generalizations," Applied Mathematics and Computation, vol. 199, no. 2, pp. 464-481, 2008.

[14] M. De La Sen and S. Alonso-Quesada, "Model-matching-based control of the Beverton-Holt equation in ecology," Discrete Dynamics in Nature and Society, vol. 2008, Article ID 793512, 21 pages, 2008.

[15] M. De la Sen and S. Alonso-Quesada, "Control issues for the Beverton-Holt equation in ecology by locally monitoring the environment carrying capacity: non-adaptive and adaptive cases," Applied Mathematics and Computation, vol. 215, no. 7, pp. 2616-2633, 2009.

[16] M. De La Sen and S. Alonso-Quesada, "On vaccination control tools for a general SEIRepidemic model," in 18th IEEE Mediterranean Conference on Control and Automation (MED '10), pp. 1322-1328, June 2010.

[17] M. De la Sen and S. Alonso-Quesada, "Vaccination strategies based on feedback control techniques for a general SEIR-epidemic model," Applied Mathematics and Computation, vol. 218, no. 7, pp. 3888-3904, 2011.

[18] M. De la Sen, A. Ibeas, S. Alonso-Quesada, and R. Nistal, “On the equilibrium points, boundedness and positivity of a SVEIRS epidemic model under constant regular constrained vaccination," Informatica, vol. 22, no. 3, pp. 339-370, 2011.

[19] P. van den Driessche and J. Watmough, "Reproduction numbers and sub-threshold endemic equilibria for compartmental models of disease transmission," Mathematical Biosciences, vol. 180, pp. 29-48, 2002.

[20] R. A. Horn and C. R. Johnson, Topics in Matrix Analysis, Cambridge University Press, Cambridge, UK, 1991.

[21] A. Korobeinikov and G. C. Wake, "Lyapunov functions and global stability for SIR, SIRS, and SIS epidemiological models," Applied Mathematics Letters, vol. 15, no. 8, pp. 955-960, 2002.

[22] A. Korobeinikov, "Lyapunov functions and global properties for SEIR and SEIS epidemic models," Mathematical Medicine and Biology, vol. 21, no. 2, pp. 75-83, 2004.

[23] A. Korobeinikov, "Global properties of SIR and SEIR epidemic models with multiple parallel infectious stages," Bulletin of Mathematical Biology, vol. 71, no. 1, pp. 75-83, 2009.

[24] A. Korobeinikov, "Global properties of basic virus dynamics models," Bulletin of Mathematical Biology, vol. 66, no. 4, pp. 879-883, 2004.

[25] P. Georgescu and Y.-H. Hsieh, "Global stability for a virus dynamics model with nonlinear incidence of infection and removal," SIAM Journal on Applied Mathematics, vol. 67, no. 2, pp. 337-353, 2006.

[26] A. Korobeinikov and P. K. Maini, "Non-linear incidence and stability of infectious disease models," Mathematical Medicine and Biology, vol. 22, no. 2, pp. 113-128, 2005.

[27] A. Korobeinikov, "Global properties of infectious disease models with nonlinear incidence," Bulletin of Mathematical Biology, vol. 69, no. 6, pp. 1871-1886, 2007.

[28] A. Korobeinikov, "Lyapunov functions and global stability for SIR and SIRS epidemiological models with non-linear transmission," Bulletin of Mathematical Biology, vol. 68, no. 3, pp. 615-626, 2006.

[29] J. K. Hale, Ordinary Differential Equations, Wiley-Interscience, New York, NY, USA, 1969.

[30] E. A. Barbashin, Introduction to the Theory of Stability, Wolters-Noordhoff, Groningen, The Netherlands, 1970.

[31] J. La Salle and S. Lefschetz, Stability by Liapunovs Direct Method, Academic Press, New York, NY, USA, 1961. 


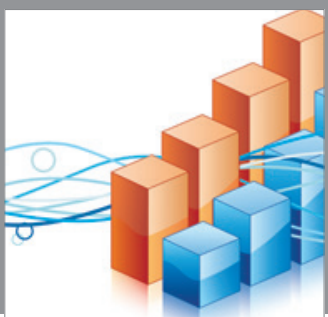

Advances in

Operations Research

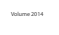

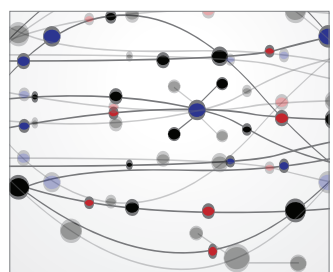

\section{The Scientific} World Journal
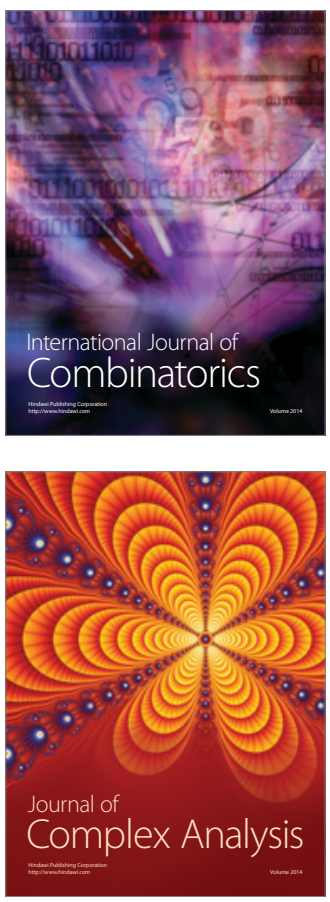

International Journal of

Mathematics and

Mathematical

Sciences
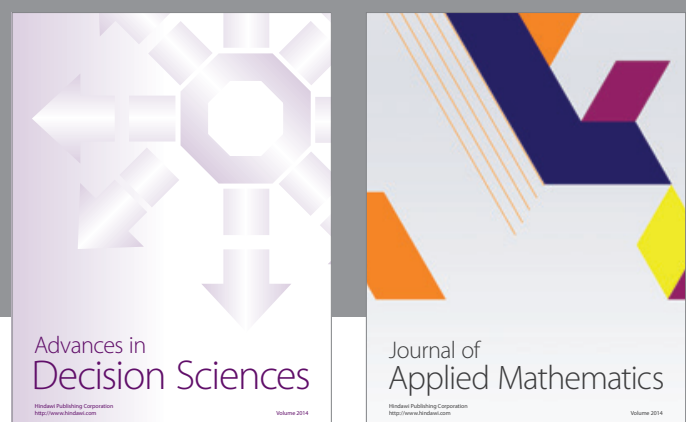

Journal of

Applied Mathematics
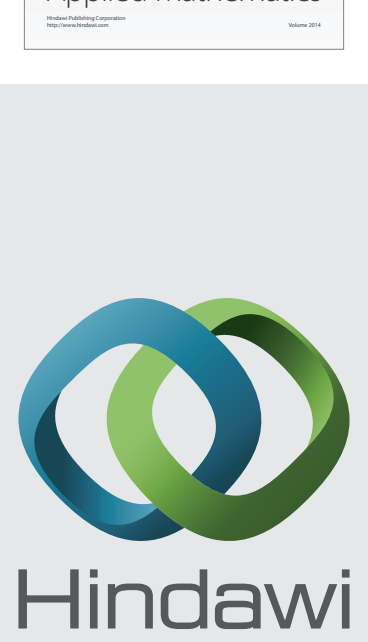

Submit your manuscripts at http://www.hindawi.com
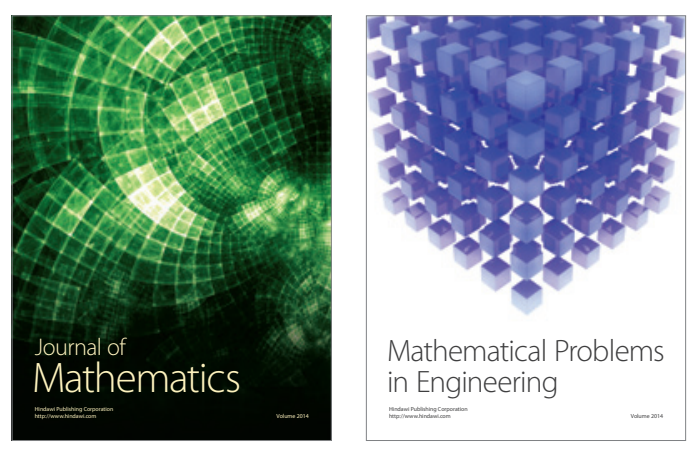

Mathematical Problems in Engineering
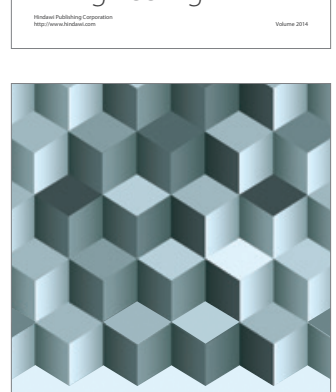

Journal of

Function Spaces
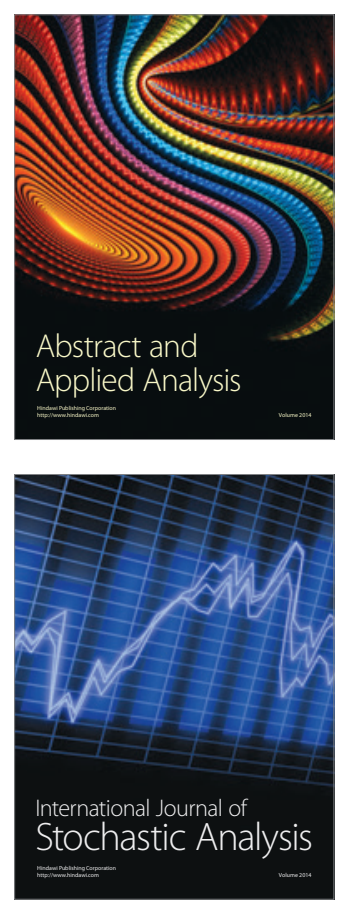

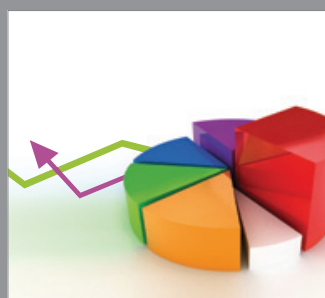

ournal of

Probability and Statistics

Promensencen
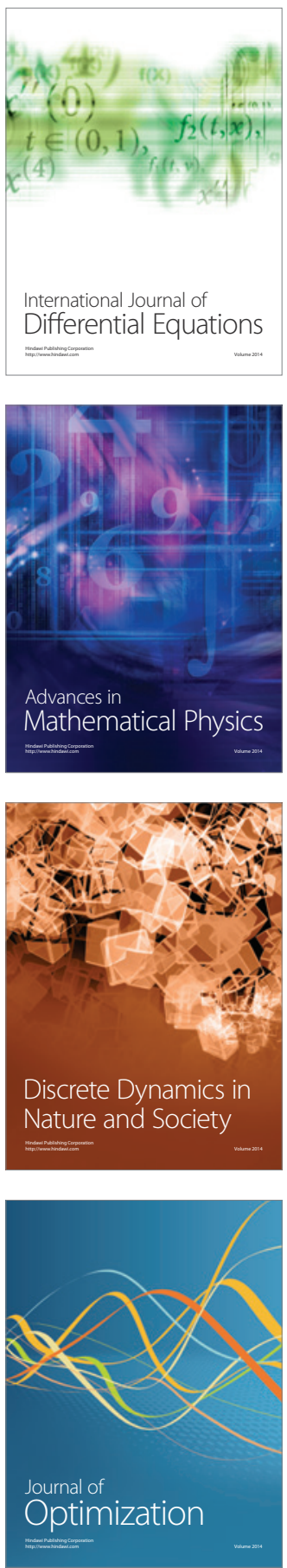